\title{
Willis Metamaterial on a Structured Beam
}

\author{
Yongquan Liu, ${ }^{1,2}$ Zixian Liang, ${ }^{3}$ Jian Zhu, ${ }^{2}$ Lingbo Xia,,${ }^{2,4}$ Olivier Mondain-Monval, ${ }^{5}$ \\ Thomas Brunet, ${ }^{6}$ Andrea Alù,,"* and Jensen $\mathrm{Li}^{1,2, \dagger}$ \\ ${ }^{1}$ Department of Physics, The Hong Kong University of Science and Technology, \\ Clear Water Bay, Hong Kong, China \\ ${ }^{2}$ School of Physics and Astronomy, University of Birmingham, Birmingham B15 2TT, United Kingdom \\ ${ }^{3}$ College of Electronic Science and Technology, Shenzhen University, Shenzhen 518060, China \\ ${ }^{4}$ Photonics Initiative, Advanced Science Research Center, City University of New York, \\ New York, New York 10031, USA \\ ${ }^{5}$ University of Bordeaux, CNRS, UMR 5031, CRPP, 33600 Pessac, France \\ ${ }^{6}$ University of Bordeaux, CNRS, UMR 5295, I2M-APy, 33405 Talence, France
}

(Received 20 July 2018; revised manuscript received 14 December 2018; published 28 February 2019)

\begin{abstract}
Bianisotropy is common in electromagnetism whenever a cross-coupling between electric and magnetic responses exists. However, the analogous concept for elastic waves in solids, termed as Willis coupling, is more challenging to observe. It requires coupling between stress and velocity or momentum and strain fields, which is difficult to induce in non-negligible levels, even when using metamaterial structures. Here, we report the experimental realization of a Willis metamaterial for flexural waves. Based on a cantilever bending resonance, we demonstrate asymmetric reflection amplitudes and phases due to Willis coupling. We also show that, by introducing loss in the metamaterial, the asymmetric amplitudes can be controlled and can be used to approach an exceptional point of the non-Hermitian system, at which unidirectional zero reflection occurs. The present work extends conventional propagation theory in plates and beams to include Willis coupling and provides new avenues to tailor flexural waves using artificial structures.
\end{abstract}

DOI: 10.1103/PhysRevX.9.011040

\section{INTRODUCTION}

Metamaterials, constructed with artificially designed microstructures, have been employed and developed in electromagnetism [1,2], acoustics [3-7], thermodynamics $[8,9]$, and mechanics [10-12] to give unique properties beyond those provided by natural and composite materials. Such a concept has recently been extended to elastic waves in solids [13-17]. Compared to acoustic waves, the additional degrees of freedom in polarizations states require more sophisticated dispersion engineering [18-20] and also lead to nontrivial mode matching at interfaces. Metamaterials are found particularly useful as a tool to explore the physics behind this complexity. For example, for in-plane waves, a transmodal Fabry-Pérot (FP) condition is found necessary for maximum mode conversion

\footnotetext{
* Corresponding author. aalu@gc.cuny.edu

Corresponding author. jensenli@ust.hk

Published by the American Physical Society under the terms of the Creative Commons Attribution 4.0 International license. Further distribution of this work must maintain attribution to the author(s) and the published article's title, journal citation, and DOI.
}

Subject Areas: Acoustics, Metamaterials

between longitudinal and shear modes [21]. For flexural waves [22,23], evanescent modes can be used to modify surface impedance for obtaining higher transmission than structures in acoustics [24].

Peculiarly, classical elastic wave equations, i.e., Hooke's law together with Newton's second law, are not form invariant under a spatial coordinate transformation [25,26], suggesting that we are currently exploring an unnecessarily limited palette of material properties. For example, an introduction of rotation modulus in Hooke's law [27] is found necessary to describe biological composites such as wet bones [28]. This is called a Cosserat solid or a micropolar medium and has been recently constructed using a metamaterial approach [29]. Another attempt is the proposal to realize Willis media [30]. These media introduce new constitutive terms not only in Hooke's law but also in Newton's second law [31-35]. These terms, which introduce coupling between stress and velocity and between momentum and strain, are typically small perturbations and are difficult to realize. Currently, a similar modification to both equations was experimentally introduced in airborne acoustics [36-38], and a strategy to induce strong Willis coupling in suitably designed acoustic metamaterials was theoretically introduced [39]. The acoustic wave equations, when Willis coupling is considered, can be written in analogy to the electromagnetic scenario, and the additional constitutive 
terms correspond to bianisotropy in electromagnetism [40-43]. These developments indicate that Willis media for elastic waves in solid may become practical through the notion of metamaterials, although the exact microstructural design has yet to be determined.

In this work, we consider the situation when a Willis medium is used to construct a plate or a beam for flexural wave propagation. Such a reduced version from three to two dimensions facilitates analysis and an intuitive understanding of Willis coupling. Plates and beams are actually common in a wide range of length scales from building structures to micromechanical systems. Their theories can be traced back to the 1950s to 1980s in a series of works from Kirchhoff-Love plate theory to extensions with rotary and shear deformations, and from isotropic to anisotropic plates and beams $[44,45]$. However, further extension to include Willis coupling to plate or beam theory is deemed necessary to open new directions in this field of research. The special responses offered by artificially tunable Willis coupling, including asymmetric reflection amplitudes and exceptional points, open an unexplored territory for ultrasonic nondestructive evaluation, seismic metamaterials for earthquake protection, and microsensor technology [14,22,46-48].

\section{WILLIS PLATE AND BEAM THEORY}

We start from a Willis medium, with a constitutive relation in its most general form [33]:

$$
\begin{aligned}
\sigma_{i j} & =C_{i j k l} \varepsilon_{k l}-\omega^{2} S_{i j k} u_{k}, \\
p_{j} & =-i \omega S_{k l j} \varepsilon_{k l}-i \omega \rho_{i j} u_{i},
\end{aligned}
$$

where $C$ and $\rho$ are the stiffness and density tensors, $\omega$ is the radial frequency, and $i, j, k$ are indices iterating the spatial coordinates. The additional $S$ term in Hooke's law, the Willis coupling coefficient, couples stress $\sigma$ to displacement $u$, and the same term (due to reciprocity) in Newton's second law couples momentum $p$ to strain $\varepsilon$. Suppose that we now construct a plate $(z=-h / 2$ to $h / 2)$ from this medium. By integrating Eq. (1) along $z$ (zeroth moment), we have

$$
\begin{aligned}
& Q_{x}=2 \mu h \varepsilon_{x z}-\omega^{2} h \tau u_{z}, \\
& P_{z}=-2 i \omega h \tau \varepsilon_{x z}-i \omega \rho_{z z} h u_{z} .
\end{aligned}
$$

We assume flexural wave propagation along the $x$ direction $\left(\partial_{y} \rightarrow 0, u_{y}=0\right)$. Equation (2) relates the shear force $Q_{x}=\int \sigma_{x z} d z$ and the total momentum $P_{z}=\int p_{z} d z$ to $u_{z}$ and $\varepsilon_{x z} . \mu$ and $\rho_{z z}$ are recognized as the effective shear modulus $\left(C_{x z x z}\right.$ in the bulk) and density along $z$ for the plate. The additional constant $\tau$ (it is actually $S_{x z z}$ in the bulk) is a Willis coupling term. For plate theory, we actually need to integrate up to the first moment $\left(\int z d z\right)$ of Eq. (1) and the equation of motion, yielding
$\partial_{x}^{2}\left(D \partial_{z} \varepsilon_{x x}\right)+\frac{h^{3} \omega^{2}}{12} \partial_{x}\left(\varrho_{x x} \partial_{z} u_{x}\right)=\partial_{x} Q_{x}=-i \omega P_{z}$,

which has the same form in Mindlin plate theory [44], but now with Willis coupling terms in $Q_{x}$ and $P_{z}$ to describe propagation of $u_{x}$ and $u_{z}$. D is the bending stiffness of the plate [49]. The second term involving $\varrho_{x x}$, the effective density along $x$, refers to rotary motion, which can be neglected when the plate is sufficiently thin. Furthermore, if we use the Willis material to construct a narrow beam (confining the width of the plate in the $y$ direction), Eq. (2) stays exactly the same with the same values of coefficients while Eq. (3) keeps the same form but with the values of $D$ and $\varrho_{x x}$ renormalized from a plate to a beam version (see Appendix A for more details). Therefore, Eqs. (2) and (3) describe wave propagation along the $x$ direction for a Willis plate or beam although we have not yet designed the mechanism to generate the required Willis coupling. We note that there is another Willis coupling term in the first moment [which will appear inside the two brackets in Eq. (3)], but its effect is much smaller in our designed metamaterial; see the $\kappa$ term for details in Appendix A.

\section{METAMATERIAL DESIGN WITH WILLIS COUPLING}

As the Willis coupling term $\tau$ comes from $S_{x z z}$ of the bulk, a necessary condition to have nonzero $\tau$ is broken mirror symmetry in the $x$ direction. Figure 1(a) shows the unit cell of the metamaterial plate designed by perforating slots in a background acrylic plate. The inner disk is connected to the matrix by two thin ribs, either along the $y$ direction (as a reference case without Willis coupling in the next section) or both connected to the back interface (as in the case with Willis coupling). Various dimensions of the structure are listed in the caption. In the latter case, there is a cantilever bending resonance for the central disk at around $14.5 \mathrm{kHz}$ [50], designed to implement a strong Willis coupling: when there is a constant force $F_{z}$ applied along the $z$ direction on both the front and back interfaces, a typical simulated displacement profile $u_{z}$ around the resonating frequency is shown as the color map. The back interface, being dragged by the disk, has a smaller magnitude of displacement than the front interface. This asymmetry gives rise to nonzero Willis coupling between $P_{z}$ (equivalent to $F_{z}$ ) and $\varepsilon_{x z}$ in Eq. (2).

This asymmetry of the displacement field is connected to asymmetric reflection. Suppose that 5 unit cells are cascaded and embedded in the background beam. The forward and backward reflection amplitudes, $r_{f}$ and $r_{b}$, are obtained by full-wave simulations (finite element method) from 7 to $12 \mathrm{kHz}$ (corresponding to wavelengths from 28.1 to $21.2 \mathrm{~mm}$ ) and are plotted in Fig. 1(b). As the metamaterial is reciprocal, we have equal transmission $t$ in both directions and, hence, the same magnitude of reflection amplitude if the material is lossless: $\left|r_{f}\right|^{2}=1-|t|^{2}=\left|r_{b}\right|^{2}$. The asymmetry 
(a)

Symmetric material

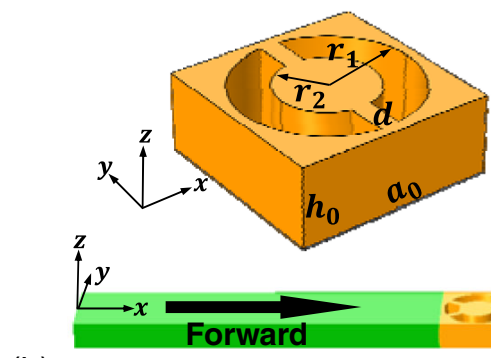

(b)

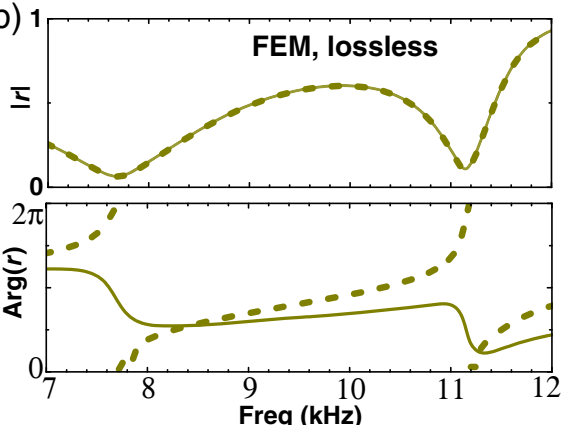

(d)

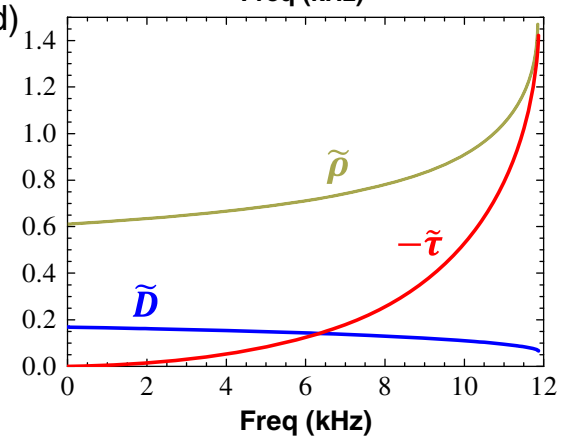

Willis material

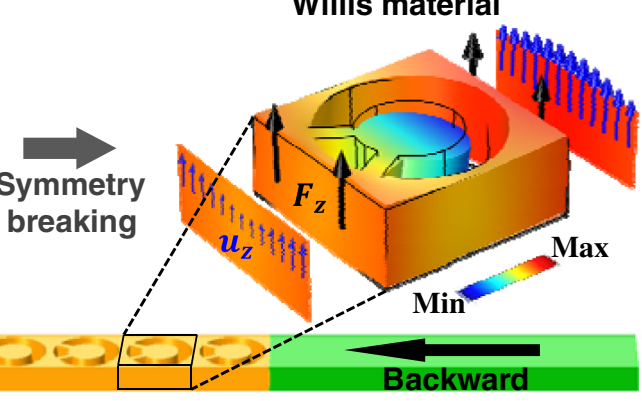

(c)

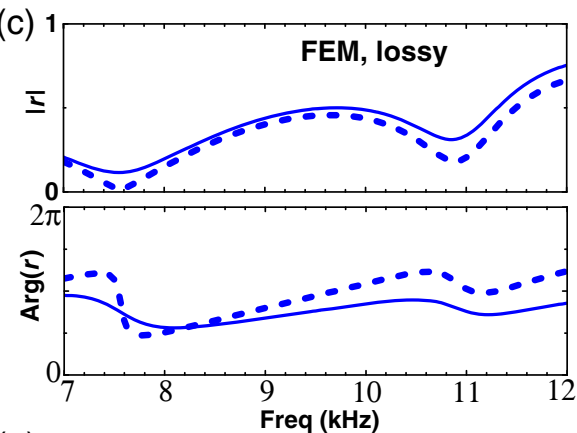

(e)
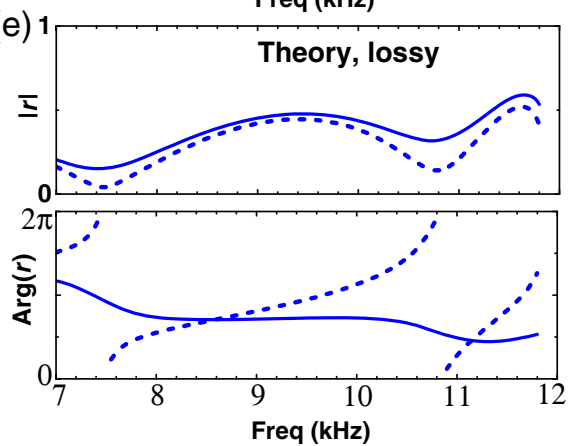

FIG. 1. Symmetry breaking in reflection and Willis metamaterial. (a) Symmetric metamaterial is changed into the Willis one by breaking mirror symmetry. For this Willis material, $a_{0}=5.0 \mathrm{~mm}, h_{0}=2.0 \mathrm{~mm}, r_{1}=0.45 a_{0}, r_{2}=0.25 a_{0}$, and $d=0.5 \mathrm{~mm}$, with orientation angles of two thin ribs $150^{\circ}$ and $210^{\circ}$, respectively. The displacement field $u_{z}$ is plotted by loading force $F_{z}$ (shown as black arrows). The field of $u_{z}$ on the two end surfaces is also presented by blue arrows. The Willis material is assembled as a metamaterial layer in a thin beam. Forward (backward) plane wave is incident into the left (right) side of the beam. (b) Amplitudes $|r|$ and phases $\arg (r)$ of reflected waves from both sides for lossless material (density $\rho_{0}=1190 \mathrm{~kg} / \mathrm{m}^{3}$, Young's modulus $E_{0}=2.95 \mathrm{GPa}$, and Poisson ratio $\nu_{0}=0.29$ ) obtained from finite element method (FEM). (c) Amplitudes $|r|$ and phases $\arg (r)$ of reflected waves for lossy material [Young's modulus turns into $E_{0}=2.95(1-0.05 i) \mathrm{GPa}$. (d) Effective parameters at different frequencies. (e) Theoretical values of reflection amplitudes $|r|$ and phases $\arg (r)$ for lossy material obtained from the effective medium in (d). In (b), (c), and (e), the solid (dashed) lines correspond to the case of forward (backward) incident waves.

of reflection shows up only in the phase and becomes most prominent at 7.7 and $11.0 \mathrm{kHz}$, in which Fabry-Pérot resonances [51] for elastic waves occur at the reflection amplitude dips. If we add a material loss (by adding 5\% of the real part of Young's modulus to the imaginary part), the magnitude of the reflection amplitudes becomes asymmetric as well, as shown in Fig. 1(c).

The asymmetry in reflection can be understood in terms of the Willis coupling parameter. First, we define an effective medium model of our structure from the eigenmode profiles (see Appendix B for details). The normalized bending stiffness $\tilde{D}=D / D_{0}$, normalized density $\tilde{\rho}=\rho / \rho_{0}$, and normalized Willis coupling parameter $\tilde{\tau}=(\tau / \mu)\left(\omega^{2} / k_{0}\right)$ are plotted in Fig. 1(d). These are the minimal set of dimensionless parameters for our case to describe the metamaterial with an effective medium model. $D_{0}, \rho_{0}$, and $k_{0}$ are the corresponding bending stiffness, density, and wave number for the background beam. Then, the transfer matrix can be formulated numerically from the effective medium with thickness of 5 unit cells. The reflection amplitudes (for the lossy case) obtained from the effective medium are then plotted in Fig. 1(e) as theory results, showing similar behavior to full-wave simulation. In the long-wavelength limit, the transfer matrix can be simplified analytically, giving rise to a difference of reflection amplitudes $r_{f}-r_{b} \approx-\tilde{\tau} k_{0} l e^{-i k_{0} l}$, where $l$ is the total 
thickness of the metamaterial (see Appendix C for details). This provides a qualitative understanding of the contribution of the Willis coupling to asymmetric reflection. When we turn off $\tilde{\tau}$ by setting it to zero, the reflection amplitudes become the same for both directions as expected (results not shown). In addition, we obtain similar wave propagation behaviors and effective parameters for Willis materials in a structured plate [50], indicating that the present design works not only in beams but also in platelike structures.

\section{EXPERIMENTAL VERIFICATION OF WILLIS PROPERTIES}

A series of experimental studies have been performed to demonstrate Willis coupling and the resultant asymmetric reflection. Figure 2(a) shows the photographs of two experimental samples, with geometric dimensions listed in the caption. The top one shows our designed Willis material while the bottom one shows a symmetric metamaterial. Several unit cells for either structure are 3D printed (using a Stratasys Objet30 Pro 3D printer, based on photopolymerization) as a structured beam with length, width, and thickness 280.0, 5.0, and $2.0 \mathrm{~mm}$, respectively. OBJET VEROBLUE RGD840 (acrylic) is chosen as the printing material, with measured Young's modulus $E_{0}=2.95(1-0.04 i) \mathrm{GPa}$, Possion's ratio $\nu_{0}=0.29$, and density $\rho_{0}=1190 \mathrm{~kg} / \mathrm{m}^{3}$. A five-cycle tone burst with the central frequency of $10 \mathrm{kHz}$ is defined in a signal generator channel of a scanning laser vibrometer (Polytec PSV-400). After the amplification of a power amplifier (KH 7602M), the pulse signal transfers to a piezoelectric transducer to generate flexural plane waves from the left (forward) or the right (backward) directions. Blue tack is attached on both ends of each beam as absorbers to diminish reflected waves. The out-of-plane displacement field $(160 \mathrm{~mm}$ length in the central area, with a spatial resolution of $2.0 \mathrm{~mm}$ ) is scanned by a laser vibrometer. After the fast Fourier transform (FFT), the wave field at each frequency is obtained and used to further extract the transmission and reflection spectrum (see Appendix D for details).

Figure 2(b) shows the reflection amplitudes given by the Willis material, shown by the blue and red symbols for 5 and 3 unit cells, respectively. The reflection amplitude $\left(\left|r_{b}\right|\right)$ in the backward direction is smaller than the one $\left(\left|r_{f}\right|\right)$ in the forward direction. Such an asymmetry is most prominent near the FP resonances: the reflection dips at 7.6 and $11.0 \mathrm{kHz}$ for the 5-unit-cell sample, where the difference of the reflection amplitude $\Delta|r|=\left|r_{f}\right|-\left|r_{b}\right|$ reaches to local maximums 0.10 and 0.18 , and at $9.0 \mathrm{kHz}$ for the 3-unit-cell sample, where the difference reaches to 0.08 . This indicates that our system actually exhibits a certain amount of material loss (as assumed in Sec. III). The multiple reflections near the FP condition within the unit cells forces the waves to interact more strongly with the bending cantilever resonance, and, hence, the asymmetry in amplitude becomes larger. On the contrary, the gray symbols in the same figure show the forward and backward reflection amplitude for a similar structure (but symmetric), in which no asymmetric reflection occurs. The observed (a)



(d)

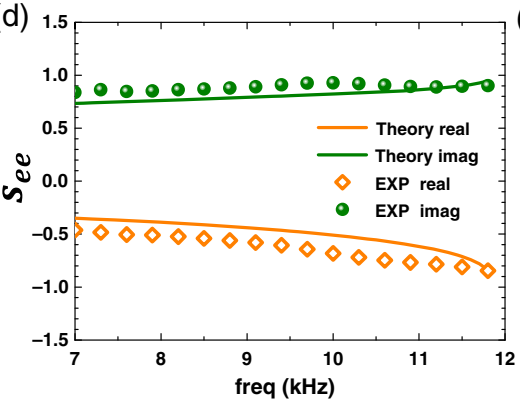

(b)
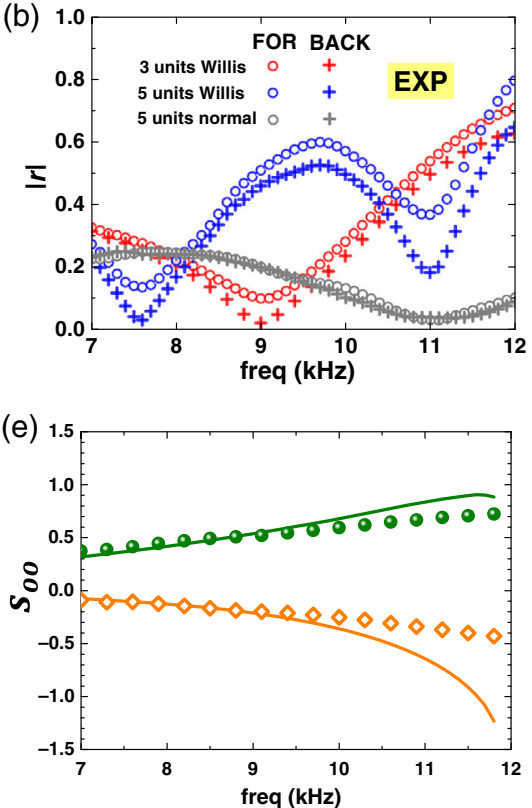
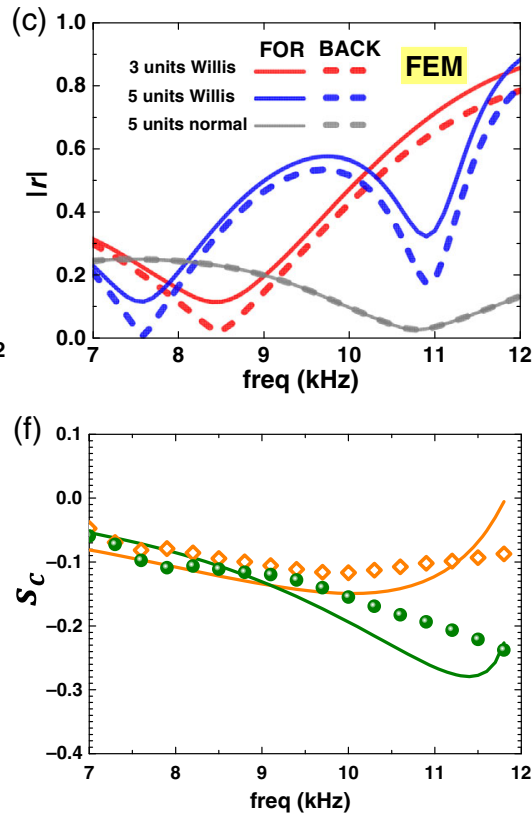

FIG. 2. Experimental testing of asymmetric reflection and extraction of Willis coupling. (a) Photograph of Willis and symmetric metamaterials. (b),(c) The experimentally tested and simulated reflected amplitude of different cases, respectively. For the symmetric material, $r_{1}=0.4 a_{0}, d=0.4 \mathrm{~mm}$, orientation angles of the two thin ribs being set along the $y$ direction. Other geometric and material parameters are same as the Willis case. (d)-(f) The theoretical (solid lines) and experimental (symbols) values of the even scattering coefficient $s_{e e}$, the odd scattering coefficient $s_{o o}$, and the cross-coupling scattering coefficient $s_{c}$ of the Willis metamaterial. 
asymmetry in reflection also agrees well with full-wave simulation results, shown in Fig. 2(c). For example, the Willis material with 3 unit cells exhibits the same FP dip at $8.6 \mathrm{kHz}$ with quantitative agreement $\Delta|r|=0.09$ to the experiment.

As we have measured the complex transmission and reflection coefficients for excitation from both sides, we can reveal Willis coupling directly by plotting the even and odd scattering coefficients. Similar to the case of electromagnetic metamaterials [52,53], materials without bianisotropy only convert even (odd) inputs to even (odd) scattered waves (termed as $s_{e e}$ and $s_{o o}$ ). The Willis coupling here contributes a cross-coupling between even input and odd output or equivalently between odd input and even output (termed as $s_{c}$, it represents $s_{e o}=-s_{o e}$ with the same amplitude but a minus sign from reciprocity [32,33]). Figures 2(d)-2(f) show the even scattering coefficient $s_{e e}$, the odd scattering coefficient $s_{o o}$. and the cross-coupling scattering coefficient $s_{c}$. First, the scattering matrix (parameters $t, r_{f}$, and $r_{b}$ ) can be either measured from experiment for a beam sample with only one unit cell or obtained from theory: the scattering matrix resultant from the effective medium in Fig. 1(d). Then, the even and odd scattering coefficients can be obtained by linearly combining the (a)

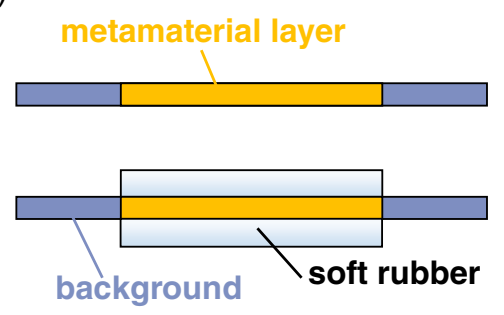

(c)

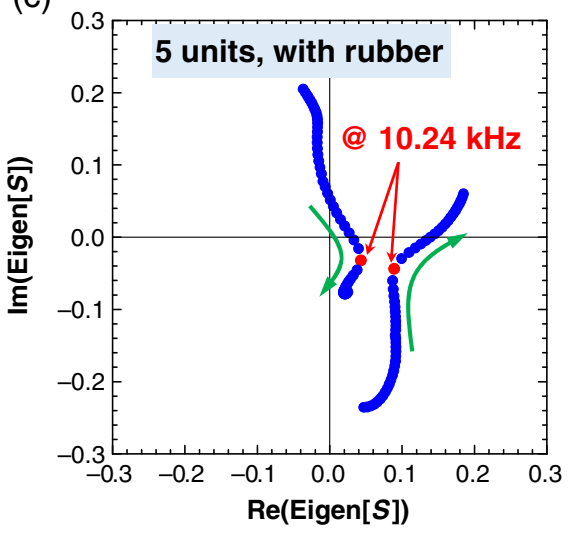

(e)

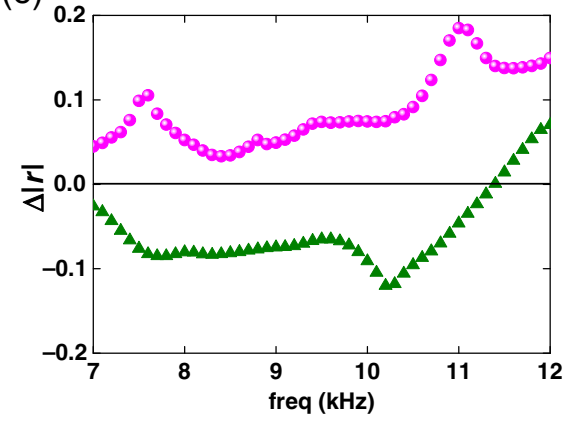

(b)

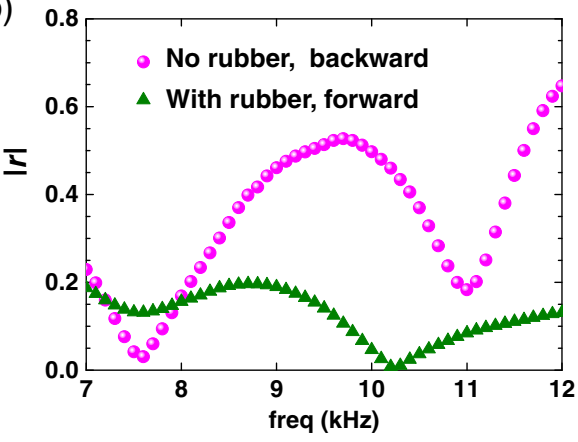

(d)

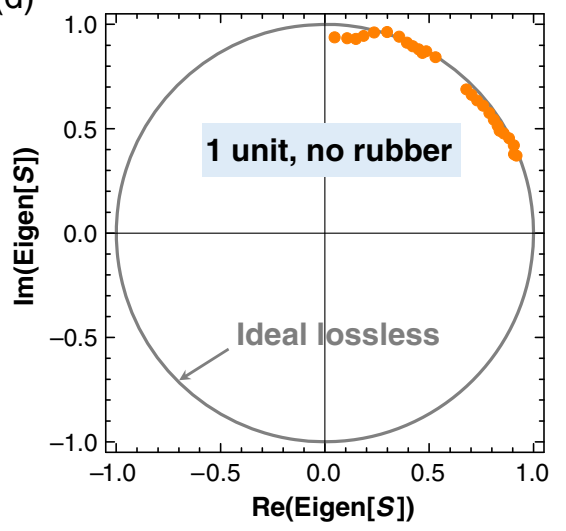

(f)

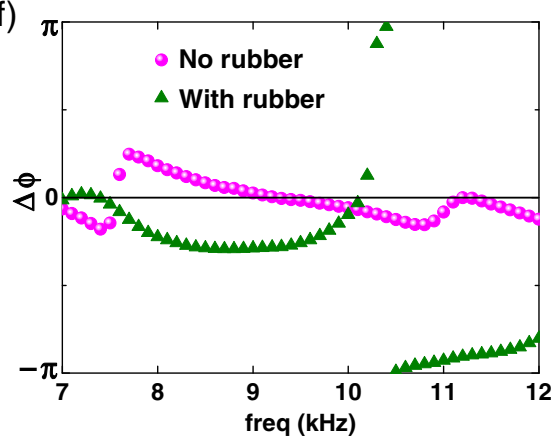

FIG. 3. Tunable asymmetry by loading lossy porous rubber. (a) Schematic of adding loss by attaching soft porous rubber patches to the metamaterial layer. (b) Measured amplitudes of the reflected waves $|r|$ with and without porous rubber patches. (d) Eigenvalues of the $S$ matrix at different frequencies for a 5-unit Willis metamaterial layer, with soft porous rubber patches attached on surfaces. Green arrows represent the trajectories of both eigenvalues along with the increase of the frequency. (d) Eigenvalues of the $S$ matrix at different frequencies for a 1-unit Willis metamaterial layer, no soft porous rubber pasted. The case of ideal lossless layer is a perfect unit circle. (e) Amplitude difference of reflected waves $\Delta|r|=\left|r_{f}\right|-\left|r_{b}\right|$. (f) Phase difference of reflected waves $\Delta \phi=A r g\left(r_{f} / r_{b}\right)$ for both cases. 
matrix elements (see Appendix E for details). In our case, a nonzero cross-coupling scattering coefficient $s_{c}$ [approximated by $-0.1+(0.24-0.04 f) i$, with $f$ being the frequency in $\mathrm{kHz}$ ] directly reveals the effective "bianisotropy" given by Willis materials. Moreover, due to symmetry breaking, the bending cantilever resonance can now be excited by both even and odd inputs, giving rise to $s_{o o}$ and $s_{e e}$. On the contrary, $s_{c}$ becomes zero if a mirror symmetric structure is used for testing.

\section{TUNABLE ASYMMETRY BY VARYING THE LOSS COEFFICIENT}

Next, we show that the degree of asymmetry and also the frequencies (the FP dips) where the asymmetry occurs can be easily tuned by loading additional materials with loss to the Willis metamaterial. Here, soft porous silicon rubber synthesized by thermal polymerization [54] is pasted onto the upper and lower surfaces of the metamaterial layer (with 5 unit cells), as schematically shown in Fig. 3(a). Figure 3(b) shows the experimental reflection amplitudes for metamaterials with soft porous rubbers (with thickness of $2 \mathrm{~mm}$ and porosity of 12\%). Apart from tuning the loss, the pasted porous rubber largely changes the band structure and eigenmodes of the metamaterial, which leads to a different set of effective material parameters. As a result, a FP dip is observed at $10.24 \mathrm{kHz}$. Furthermore, when an appropriate amount of loss is added to the metamaterial, the reflection dip actually approaches zero (for one direction), yielding the so-called unidirectional zero reflection (UZR) [50]. For the case with two porous rubber sheets loaded, the reflection dip in the forward direction attains a value of 0.004 , very close to zero, while the reflection amplitude in the other direction is around 0.128 . The quality of the UZR can also be visualized by examining the eigenvalues of the scattering matrix $S$ (truncated to $2 \times 2$ for propagating modes) in the complex plane. For a non-Hermitian system, like the one at hand, the eigenvalue degeneracy of the scattering matrix is called the exceptional point of the system and a UZR occurs exactly at that point [53]. As shown in Fig. 3(c), trajectories against the frequency for both eigenvalues of $S$ first move towards each other, approaching the exceptional point at $10.24 \mathrm{kHz}$, and then split again with increasing frequency. If we can tune the loss in a finer degree than the current experiment, it is possible to further improve the quality by bringing the two trajectories even closer to each other [50]. On the contrary, we also plot the eigenvalues of $S$ for the case with only one unit cell (without the porous rubber sheet loading) in Fig. 3(d). In this case, loss is reduced, and it does not support FP resonances to create large asymmetry in reflection amplitudes. In such a case, the eigenvalues are nondegenerate and are located near the unit circle corresponding to the lossless case.

In fact, the loading of porous rubber sheets can be an effective way to tune not only the UZR frequency position but also the degree of reflection asymmetry, on both the amplitude difference $\Delta|r|=\left|r_{f}\right|-\left|r_{b}\right|$ and the phase difference $\Delta \phi=\arg \left(r_{f} / r_{b}\right)$. Figure 3(e) shows the measured $\Delta|r|$, which changes from positive to negative by the additional loading of porous rubber sheets. Such a change occurs over a wide frequency bandwidth as well. Figure 3(f) shows the phase difference between the two directions. $\Delta \phi$ becomes large and can cover the whole $2 \pi$ range. This is due to the current employment of the FP resonance in creating the asymmetry combined with the UZR (the most extreme asymmetry from an exceptional point) for the two-sheets case, different from the demonstrated airborne-acoustic bianisotropy with $\Delta \phi<\pi / 6$ [37], and further illustrates a nontrivial feature of our lossy Willis materials.

\section{DISCUSSION}

By extending conventional plate and beam theory with the introduction of Willis materials, we have designed and experimentally realized effective bianisotropy in a Willis metamaterial for elastic waves using a cantilevertype resonating structure. The metamaterial induces cross-coupling between shear force and vertical displacement of the plate, or equivalently between vertical momentum and shear strain. The present design works for flexural waves on both platelike and beamlike structures. The effective medium parameters, including the Willis coupling, are confirmed through experimental measurements of the asymmetric reflection amplitudes in the forward and backward propagation directions. Unlike previous studies on acoustic bianisotropic materials, not only asymmetric phases but also asymmetric amplitudes of reflection are observed due to the presence of loss, whose effect is further magnified through a combination of the cantilever bending resonance and a FP resonance. Interestingly, by incorporating an appropriate amount of loss, we have demonstrated the most asymmetric case in which the reflection amplitude goes to zero in one direction. Unidirectional zero reflection is achieved when approaching the exceptional point of the scattering matrix if the metamaterial is interpreted as a non-Hermitian system. Together with the demonstrated tunability of the responses (asymmetric reflection amplitudes, phases, and frequency dips), the proposed metamaterials operating at the exceptional point can be applied to realize high- $Q$ sensors [55,56], tunable metasurfaces, and asymmetric wave front control [57]. Elastic bianisotropy for flexural waves can be generalized to Lamb waves for intermodal conversion control, to Rayleigh waves in seismology for earthquake protection of civil buildings, or to obtain high transmission efficiency $[14,46,58-61]$. The demonstration of a Willis metamaterial can also be used as a typical prototype to design transformation elastic devices, as for its original motivation, to construct an elastic wave cloak based on form-invariant transformations of elastic wave equations $[25,46,62]$. 


\section{ACKNOWLEDGMENTS}

J.L. acknowledges support from the Research Grants Council in Hong Kong (Grant No. 16302218). Y. L. acknowledges the financial support by the fellowship of future scientist from the China Scholarship Council (CSC). A. A. acknowledges support from the National Science Foundation. We also acknowledge support from the European Union's project NOCTURO for the visit of L. X., Z. L. acknowledges the financial support by NSFC (11574217 and 61505114).

\section{APPENDIX A: FIRST-ORDER WILLIS PLATE AND BEAM THEORY}

When a Willis medium, with $\mathscr{M}_{y}$ and $\mathscr{M}_{z}$ mirror symmetry, is in the form of a thin plate of thickness $h$, we take $\sigma_{z z} \cong 0$. Equation (1) can then be rewritten as.

$$
\begin{aligned}
\left(\begin{array}{c}
\sigma_{x z} \\
\sigma_{y z}
\end{array}\right) & =\left(\begin{array}{cc}
C_{x z x z} & 0 \\
0 & C_{y z y z}
\end{array}\right)\left(\begin{array}{c}
2 \varepsilon_{x z} \\
2 \varepsilon_{y z}
\end{array}\right)-\omega^{2}\left(\begin{array}{c}
S_{x z z} \\
0
\end{array}\right) u_{z}, \\
p_{z} & =-i \omega\left(S_{x z z} 2 \varepsilon_{x z}+\rho_{z z} u_{z}\right), \\
\left(\begin{array}{c}
\sigma_{x x} \\
\sigma_{y y} \\
\sigma_{x y}
\end{array}\right) & =\left[C^{(p)}\right]\left(\begin{array}{c}
\varepsilon_{x x} \\
\varepsilon_{y y} \\
2 \varepsilon_{x y}
\end{array}\right)-\omega^{2}\left[S^{(p)}\right]\left(\begin{array}{c}
u_{x} \\
u_{y}
\end{array}\right), \\
\left(\begin{array}{c}
p_{x} \\
p_{y}
\end{array}\right) & =-i \omega\left\{\left[S^{(p)}\right]^{T}\left(\begin{array}{c}
\varepsilon_{x x} \\
\varepsilon_{y y} \\
2 \varepsilon_{x y}
\end{array}\right)+\left[\rho_{T}^{(p)}\right]\left(\begin{array}{l}
u_{x} \\
u_{y}
\end{array}\right)\right\},
\end{aligned}
$$

where $\left[C^{(p)}\right],\left[S^{(p)}\right]$, and $\left[\rho_{T}^{(p)}\right]$ have matrix elements renormalized from the bulk constitutive parameters to the plate ones [indicated by superscript $(p)$ ]. For a thin plate, we only need to consider up to the first two moments of the above equations. Integrating the first two equations in Eq. (A1) by $\int d z$ and integrating the last two equations by $\int z d z$ gives Eq. (2) and

$$
\begin{aligned}
M_{x x} & =D \partial_{z} \varepsilon_{x x}-\omega^{2} \frac{h^{3}}{12} \kappa \partial_{z} u_{x}, \\
N_{x} & =-\frac{i \omega h^{3}}{12} \kappa \partial_{z} \varepsilon_{x x}-\frac{i \omega h^{3}}{12} \varrho_{x x} \partial_{z} u_{x},
\end{aligned}
$$

where $M_{x x}=\int z \sigma_{x x} d z, N_{x}=\int z p_{x} d z, \kappa=S_{x x x}^{(p)}, \varrho_{x x}=\rho_{x x}^{(p)}$, and $D=h^{3} C_{x x x x}^{(p)} / 12$ is called the bending stiffness. The coefficients $C_{x x x x}^{(p)}, S_{x x x}^{(p)}$, and $\rho_{x x}^{(p)}$ for the plate can be expressed in terms of the bulk parameters through

$$
\begin{aligned}
C_{x x x x}^{(p)} & =C_{x x x x}-\left(C_{x x z z}\right)^{2} / C_{z z z z}, \\
S_{x x x}^{(p)} & =S_{x x x}-C_{x x z z} S_{z z x} / C_{z z z z}, \\
\rho_{x x}^{(p)} & =\rho_{x x}+\omega^{2}\left(S_{z z x}\right)^{2} / C_{z z z z} .
\end{aligned}
$$

We have also used $\partial_{\mathrm{y}} \rightarrow 0$ and $u_{y}=0$ in writing Eqs. (2) and (A2) as the constitutive relationship for a flexural wave propagating along the $x$ direction on the plate. We note that in deriving Eq. (A2), we have taken the approximation that the field quantities being odd functions in $z$ are linear in $z$. On the other hand, the even (in-z) field quantities are assumed to be constant in $z$. It can be further improved by using a quadratic function in $z$ so that an additional shear correction multiplication factor appears in front of $\mu$, for example. Here, we have not employed such a higher-order correction, for brevity, as it will not affect the form.

If the Willis medium is in the form of a narrow beam of width $b$ in the $y$ direction, Eq. (A1) is further modified by taking $\sigma_{y y} \cong 0$, as

$$
\begin{aligned}
\sigma_{x z} & =C_{x z x z} 2 \varepsilon_{x z}-\omega^{2} S_{x z z} u_{z}, \\
p_{z} & =-i \omega\left(S_{x z z} 2 \varepsilon_{x z}+\rho_{z z} u_{z}\right), \\
\sigma_{x x} & =C_{x x x x}^{(b)} \varepsilon_{x x}-\omega^{2} S_{x x x}^{(b)} u_{x}, \\
p_{x} & =-i \omega\left(S_{x x x}^{(b)} \varepsilon_{x x}+\rho_{x x}^{(b)} u_{x}\right),
\end{aligned}
$$

for the flexural wave propagating on the narrow beam along the $x$ direction. The coefficients $C_{x x x x}^{(p)}, S_{x x x}^{(p)}$, and $\rho_{x x}^{(p)}$ are further renormalized from the plate to the beam version through

$$
\begin{aligned}
C_{x x x x}^{(b)} & =C_{x x x x}^{(p)}-\left(C_{x x y y}^{(p)}\right)^{2} / C_{y y y y}^{(p)}, \\
S_{x x x}^{(b)} & =S_{x x x}^{(p)}-C_{x x y y}^{(p)} S_{y y x}^{(p)} / C_{y y y y}^{(p)}, \\
\rho_{x x}^{(b)} & =\rho_{x x}^{(p)}+\omega^{2}\left(S_{y y x}^{(p)}\right)^{2} / C_{y y y y}^{(p)} .
\end{aligned}
$$

The first two moments of Eq. (A3) then give the constitutive relationship for the beam: exactly the same as Eq. (2) and another equation of the same form as Eq. (A2) except $\kappa=S_{x x x}^{(b)}, \quad \varrho_{x x}=\rho_{x x}^{(b)}, \quad D=h^{3} C_{x x x x}^{(b)} / 12$ being changed from the plate to the beam version. For isotropic material with Young's modulus $E$ and Poisson's ratio $\nu$ as a trivial example, the above formulation gives bending stiffness as $h^{3} E / 12 /\left(1-\nu^{2}\right)$ for a plate and $h^{3} E / 12$ for a beam. Again, we have not employed shear correction factor by approximating the even field quantities to be simply constant in $y$. The form of Eq. (A2) stays the same for either a plate or a beam although the values of the coefficients change. We will use Eq. (A2) interchangeably for both a Willis plate and a Willis beam.

On the other hand, the wave equations for flexural waves along the $x$ direction on a plate (in the level of Mindlin plate theory) or along a narrow beam (in the level of Timoshenko beam theory) [44] share a common form as

$$
\begin{aligned}
\partial_{x} M_{x x} & =Q_{x}-i \omega N_{x}, \\
\partial_{x} Q_{x} & =-i \omega P_{z},
\end{aligned}
$$

regardless of whether the constitutive relationship has or does not have Willis terms. It can be obtained either by 
taking moments (in $z$ ) from the bulk wave equations or by Hamilton's principle on the plate Lagrangian $\int \mathcal{L} d z$ for a plate or $\int \mathcal{L} d y d z$ for a beam with Lagrangian density $\mathcal{L}=\left(p_{i} \partial_{t} u_{i}-\varepsilon_{i j} \sigma_{i j}\right) / 2$. It is noted that Eqs. (A2) and (A4) will lead to Eq. (3) if we omit the Willis coupling term $\kappa$. Together with the geometric relation $\varepsilon_{i j}=\frac{1}{2}\left(\partial_{j} u_{i}+\partial_{i} u_{j}\right)$, we can express the governing equations [Eqs. (2), (A2), (A4)) in a combined form as

$$
-i \Pi \partial_{x}\left(\begin{array}{c}
u_{z} \\
\partial_{z} u_{x} \\
M_{x x} \\
Q_{x}
\end{array}\right)=\left[\begin{array}{cccc}
h \omega^{2}\left(\rho_{z z}+\frac{\tau^{2} \omega^{2}}{\mu}\right) & 0 & 0 & \frac{\tau \omega^{2}}{\mu} \\
0 & \frac{h^{3} \omega^{2}}{12}\left(\varrho_{x x}+\frac{h^{3} \kappa^{2} \omega^{2}}{12 D}\right) & \frac{h^{3} \kappa \omega^{2}}{12 D} & -1 \\
0 & \frac{h^{3} \kappa \omega^{2}}{12 D} & \frac{1}{D} & 0 \\
\frac{\tau \omega^{2}}{\mu} & -1 & 0 & \frac{1}{h \mu}
\end{array}\right]\left(\begin{array}{c}
u_{z} \\
\partial_{z} u_{x} \\
M_{x x} \\
Q_{x}
\end{array}\right),
$$

with

$$
\Pi=\left[\begin{array}{cccc}
0 & 0 & 0 & -i \\
0 & 0 & -i & 0 \\
0 & i & 0 & 0 \\
i & 0 & 0 & 0
\end{array}\right]
$$

\section{APPENDIX B: EFFECTIVE MATERIAL PARAMETERS RETRIEVAL METHOD}

From Eq. (A5), we can obtain the dispersion relationship as

$$
D k^{2}+\frac{h \omega^{2}\left(\mu \rho_{z z}+\tau^{2} \omega^{2}\right)}{\rho_{z z} \omega^{2}-k^{2} \mu}=\frac{h^{3} \omega^{2} \varrho_{x x}}{12},
$$

where $k$ is the wave number. It is noted that $\kappa$ does not go into the dispersion relationship. Based on Eq. (B1), the field of $\partial_{z} u_{x}$ can be expressed as

$$
\partial_{z} u_{x}=\alpha_{f} e^{i k x}+\beta_{f} e^{-K x}+\alpha_{b} e^{-i k x}+\beta_{b} e^{K x},
$$

where $\alpha_{f} / \alpha_{b}$ and $\beta_{f} / \beta_{b}$ are the amplitudes of the propagating and evanescent waves in the forward and backward direction, respectively, and

$$
k^{2}=\frac{\omega \sqrt{\Gamma}+\Delta}{24 D \mu}, \quad K^{2}=\frac{\omega \sqrt{\Gamma}-\Delta}{24 D \mu},
$$

with $\Gamma=576 D h \mu^{2}\left(\rho_{z z}+\omega^{2} \tau^{2} / \mu\right)+\omega^{2}\left(h^{3} \mu \varrho_{x x}-12 D \rho_{z z}\right)^{2}$ and $\Delta=\left(h^{3} \mu \varrho_{x x}+12 D \rho_{z z}\right) \omega^{2}$. By neglecting the shear and rotary effects by supposing $\mu \rightarrow \infty$ and $\varrho_{x x} \rightarrow 0$ [44], we have $k=K=\sqrt[4]{\rho_{z z}^{\prime} h \omega^{2} / D}$, with $\rho_{z z}^{\prime}=\rho_{z z}+\omega^{2} \tau^{2} / \mu$. On the interface between two different media, Eq. (A5) suggests the continuity of dimensionless quantities $u_{z} k_{0}$, $\partial_{z} u_{x}, M_{x x} /\left(D_{0} k_{0}\right)$, and $Q_{x} /\left(D_{0} k_{0}^{2}\right)$, where the subscript " 0 " is the values in the background medium. Thus, the continuity matrix $M$ may be written as

$$
M=\left[\begin{array}{cccc}
\frac{i n+\tilde{\tau}}{n^{2}} & \frac{n-\tilde{\tau}}{n^{2}} & \frac{-i n+\tilde{\tau}}{n^{2}} & \frac{-n-\tilde{\tau}}{n^{2}} \\
1 & 1 & 1 & 1 \\
i n \tilde{D}-\tilde{\kappa} \tilde{D} & -n \tilde{D}-\tilde{\kappa} \tilde{D} & -i n \tilde{D}-\tilde{\kappa} \tilde{D} & n \tilde{D}-\tilde{\kappa} \tilde{D} \\
-n^{2} \tilde{D} & n^{2} \tilde{D} & -n^{2} \tilde{D} & n^{2} \tilde{D}
\end{array}\right],
$$

with the continuous value of $M\left\{\alpha_{f}, \beta_{f}, \alpha_{b}, \beta_{b}\right\}$ on the interface, where $\tilde{D}=D / D_{0}$ is dimensionless bending stiffness, $\tilde{\tau}=(\tau / \mu)\left(\omega^{2} / k_{0}\right)$ and $\tilde{\kappa}=\left[\left(\kappa h^{3}\right) /(12 D)\right]\left(\omega^{2} / k_{0}\right)$ are dimensionless Willis coupling terms, and $\tilde{\rho}=\rho_{z z}^{\prime} / \rho_{0}=$ $n^{4} \tilde{D}$ is the dimensionless density with $n=k / k_{0}$.

Equation (B4) gives us a way to calculate the effective medium parameters of the metamaterial. Specifically, the eigenmodes at different values of $k$ are obtained from fullwave simulations and the effective fields can be obtained by

$$
\begin{aligned}
u_{z} & =\frac{1}{A} \int u_{z}(y, z) d A, \\
\partial_{z} u_{x} & =\frac{1}{A} \int \partial_{z} u_{x}(y, z) d A, \\
M_{x x} & =\frac{h}{A} \int z \sigma_{x x}(y, z) d A, \\
Q_{x} & =\frac{h}{A} \int \sigma_{x z}(y, z) d A,
\end{aligned}
$$

where the integrations are performed on the cross section of the unit cell, with area $A$ and perpendicular to the wave propagation direction in $x$. Then the effective material parameters can be obtained based on Eq. (B4) by 


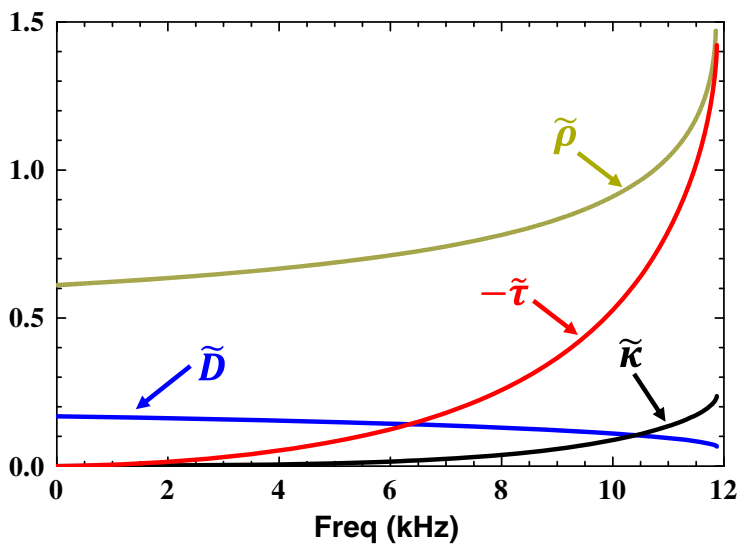

FIG. 4. Dimensionless effective parameters of the Willis metamaterial with respect to the frequency.

$$
\begin{aligned}
\tilde{D} & =\frac{\delta}{D_{0} k^{2}}, \\
\tilde{\rho} & =\frac{k^{2} \delta}{\rho_{0} h \omega^{2}}, \\
\tilde{\kappa} & =-\frac{k^{2}}{k_{0} \delta} \operatorname{Re}\left(M_{x x} / \partial_{z} u_{x}\right), \\
\tilde{\tau} & =-\frac{k^{2} \delta}{k_{0}} \frac{\operatorname{Re}\left(u_{z} / \partial_{z} u_{x}\right)}{\operatorname{Re}\left(Q_{x} / \partial_{z} u_{x}\right)},
\end{aligned}
$$

with $\delta=\sqrt{\left\{\left[\operatorname{Im}\left(M_{x x} / \partial_{z} u_{x}\right) \operatorname{Re}\left(-Q_{x} / \partial_{z} u_{x}\right)\right] /\left[\operatorname{Im}\left(u_{z} / \partial_{z} u_{x}\right)\right]\right\}}$. Figure 4 shows the simulated results of the effective parameters when Eq. (B6) is applied on a propagating eigenmode solved from COMSOL MULTIPHYSICS. In the long-wavelength limit, $\tilde{\rho} \rightarrow 0.6$ matches the analytic value given by the filling fraction. Additionally, both dimensionless Willis coupling terms $\tilde{\kappa}$ and $\tilde{\tau}$ are nearly zero at low frequencies. As expected, the amplitude of the zerothorder Willis coupling $\tilde{\tau}$ is much larger than the first-order Willis coupling $\tilde{\kappa}$ and shows significant values in the range of 7 to $12 \mathrm{kHz}$.

\section{APPENDIX C: ASYMMETRIC REFLECTION OF THE WILLIS METAMATERIAL}

Comparing the first and third columns of $M$ in Eq. (B4), we can find different eigenmodes in the positive and negative directions, leading to asymmetric wave propagation behaviors. For flexural waves incident from the background medium to a metamaterial layer with length $l$, the total transfer matrix $T$ can be written as

$$
T=M_{0}^{-1} M N M^{-1} M_{0},
$$

where $M_{0}$ is the continuity matrix of the background medium, and

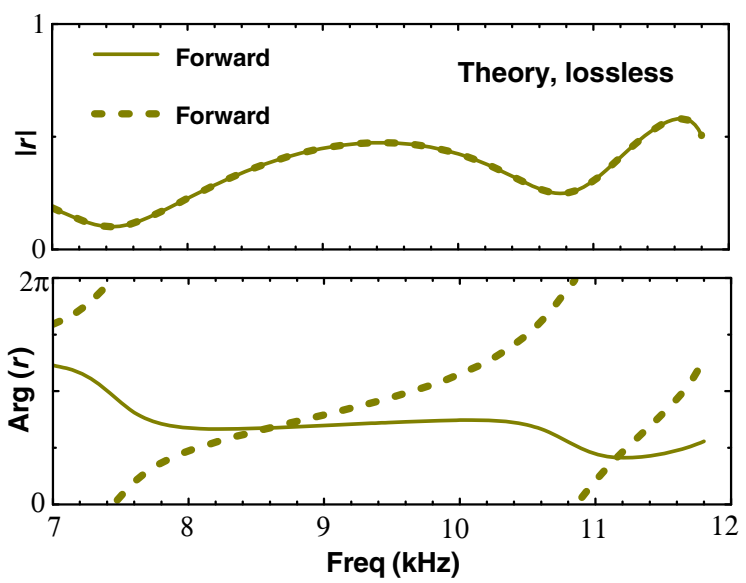

FIG. 5. Theoretical values of reflection amplitude $|r|$ and phase $\arg (r)$ from both sides for the lossless case obtained from effective medium.

$$
N=\left[\begin{array}{cccc}
e^{i k l} & 0 & 0 & 0 \\
0 & e^{-k l} & 0 & 0 \\
0 & 0 & e^{-i k l} & 0 \\
0 & 0 & 0 & e^{k l}
\end{array}\right],
$$

with $\left\{\alpha_{f, L}, \beta_{f, L}, \alpha_{b, L}, \beta_{b, L}\right\}^{T}=T\left\{\alpha_{f, R}, \beta_{f, R}, \alpha_{b, R}, \beta_{b, R}\right\}^{T}$. Subscripts $L$ and $R$ indicate the left and right sides of the system. Then the scattering matrix $\hat{S}$ in terms of $\partial_{z} u$ with $\left\{\alpha_{f, R}, \beta_{f, R}, \alpha_{b, L}, \beta_{b, L}\right\}^{T}=\hat{S}\left\{\alpha_{f, L}, \beta_{f, L}, \alpha_{b, R}, \beta_{b, R}\right\}^{T}$ can be easily obtained. If only the propagating modes are considered, the $4 \times 4$ scattering matrix $\hat{S}$ turns into a $2 \times 2$ one. Considering that $\partial_{z} u_{x} \cong-\partial_{x} u_{z}$ in a thin beam, we have $u_{z}=(i / k) \alpha_{f} e^{i k x}+(1 / K) \beta_{f} e^{-K x}-(i / k) \alpha_{b} e^{-i k x}-$ $(1 / K) \beta_{b} e^{K x}$. Thus, the $2 \times 2$ scattering matrix with respect to the displacement field $u_{z}$ should be

$$
S=\left[\begin{array}{cc}
\hat{S}_{11} & -\hat{S}_{13} \\
-\hat{S}_{31} & \hat{S}_{33}
\end{array}\right] e^{-i k_{0} l}=\left[\begin{array}{cc}
t_{f} & r_{b} \\
r_{f} & t_{b}
\end{array}\right],
$$

where the term $e^{-i k_{0} l}$ is to eliminate the reference phase by setting the origin at the center of the metamaterial layer, and $t_{f}=t_{b}$ due to the reciprocity. For a normal background medium $\tilde{\tau}_{0}=\tilde{\kappa}_{0}=0$, we have

$$
r_{f}-r_{b}=(\tilde{\kappa}-\tilde{\tau}) k_{0} l e^{-i k_{0} l} \approx-\tilde{\tau} k_{0} l e^{-i k_{0} l}
$$

in the long-wavelength limit. It is obvious that $r_{f}=r_{b}$ if the metamaterial is symmetric with $\tilde{\tau}=0$.

After obtaining the effective material parameters based on Appendix B, we theoretically calculate the reflection of the system. As shown in Fig. 5, exactly the same amplitude but different phases are observed for the lossless case. Two dips of $|r|$ are located at 7.5 and $10.8 \mathrm{kHz}$, respectively, which excellently agrees with the simulated results shown in Fig. 1(b). For the lossy case of the modulus, the material 
parameters should be changed accordingly to be complex values. As shown in Fig. 1(e), with 5\% loss of the modulus, both the reflection amplitude and phase are different now, especially around the two dips. $\left|r_{b}\right|$ is also observed much smaller than $\left|r_{f}\right|$ at about $7.5 \mathrm{kHz}$. The agreement between theoretical values and the simulated results [shown in Fig. 1(c)] shows the rationality of the present theory and the retrieved effective medium parameters.

\section{APPENDIX D: CALCULATION OF SCATTERING COEFFICIENTS}

Once the frequency-domain wave field is tested, we first suppose that the wave pattern can be described as $w_{1}=$ $\alpha e^{i k x}+\beta e^{-i k x}$ and $w_{2}=\gamma e^{i k x}+\delta e^{-i k x}$ on the incident side and the transmitted side, respectively. Here, the wave is supposed to propagate along the $x$ direction, and the origin is set at the center of the metamaterial layer. Then the complex coefficients $\alpha, \beta, \gamma$, and $\delta$ can be calculated by data fitting of the wave pattern at each frequency, for both the forward and backward case. Thus, the scattering matrix is determined by

$$
S=\left[\begin{array}{ll}
t_{f} & r_{b} \\
r_{f} & t_{b}
\end{array}\right]=\left[\begin{array}{ll}
\gamma_{f} & \gamma_{b} \\
\beta_{f} & \beta_{b}
\end{array}\right] \cdot\left[\begin{array}{cc}
\alpha_{f} & \alpha_{b} \\
\delta_{f} & \delta_{b}
\end{array}\right]^{-1},
$$

where subscripts $f$ and $b$ correspond to forward and backward incident waves, respectively.

To numerically compute the scattering matrix $S$ using COMSOL MULTIPHYSICS, perfect matched layers (PML) are used on both ends of the beam, and plane waves are generated in the forward and backward directions, respectively, to generate the field pattern. Then, similar processes as the experiments are used to calculate the scattering matrix. It is noted that the complex coefficient $\delta$ is zero due to the perfect absorption of waves by the PML. Therefore, we have $t_{f(b)}=\left[\gamma_{f(b)} / \alpha_{f(b)}\right]$ and $r_{f(b)}=\left[\beta_{f(b)} / \alpha_{f(b)}\right]$.

\section{APPENDIX E: PHYSICAL MEANING OF THE SCATTERING COEFFICIENTS}

Figure 6 illustrates the physical meaning of the scattering coefficients. In general, even (odd) inputs will lead to even $s_{e e}$ (odd $s_{o o}$ ) scattering coefficients. However, due to the symmetric breaking effects in the present work, the crosscoupling scattering coefficient $s_{c}=s_{e o}=-s_{o e}$ between even input and odd output or vice versa. Thus, we have
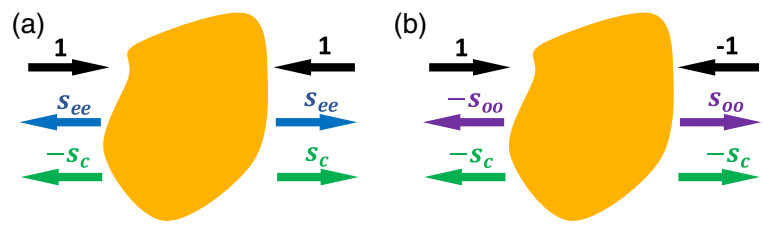

FIG. 6. Physical meaning of the scattering coefficients for the cases of (a) symmetric incident waves and (b) antisymmetric incident waves.

$$
S=\left[\begin{array}{cc}
t & r_{b} \\
r_{f} & t
\end{array}\right]=\frac{1}{2}\left[\begin{array}{cc}
2+s_{e e}+s_{o o} & s_{e e}-s_{o o}+2 s_{c} \\
s_{e e}-s_{o o}-2 s_{c} & 2+s_{e e}+s_{o o}
\end{array}\right] .
$$

Namely,

$$
\begin{aligned}
& s_{e e}=t-1+\frac{r_{b}+r_{f}}{2}, \\
& s_{o o}=t-1-\frac{r_{b}+r_{f}}{2}, \\
& s_{c}=\frac{r_{b}-r_{f}}{2} .
\end{aligned}
$$

It is readily seen that for normal metamaterials with mirror symmetry, $s_{c}=\left[\left(r_{b}-r_{f}\right) / 2\right]=0$.

[1] D. R. Smith, W. J. Padilla, D. C. Vier, S. C. Nemat-Nasser, and S. Schultz, Composite Medium with Simultaneously Negative Permeability and Permittivity, Phys. Rev. Lett. 84, 4184 (2000).

[2] V.M. Shalaev, Optical Negative-Index Metamaterials, Nat. Photonics 1, 41 (2007).

[3] Z. Liu, X. Zhang, Y. Mao, Y. Y. Zhu, Z. Yang, C. T. Chan, and P. Sheng, Locally Resonant Sonic Materials, Science 289, 1734 (2000).

[4] N. Fang, D. Xi, J. Xu, M. Ambati, W. Srituravanich, C. Sun, and X. Zhang, Ultrasonic Metamaterials with Negative Modulus, Nat. Mater. 5, 452 (2006).

[5] S. H. Lee, C. M. Park, Y. M. Seo, Z. G. Wang, and C. K. Kim, Composite Acoustic Medium with Simultaneously Negative Density and Modulus, Phys. Rev. Lett. 104, 054301 (2010).

[6] T. Brunet, A. Merlin, B. Mascaro, K. Zimny, J. Leng, O. Poncelet, C. Aristégui, and O. Mondain-Monval, Soft 3D Acoustic Metamaterial with Negative Index, Nat. Mater. 14, 384 (2015).

[7] Z. Liang and J. Li, Extreme Acoustic Metamaterial by Coiling Up Space, Phys. Rev. Lett. 108, 114301 (2012).

[8] T. Han, X. Bai, J. T. Thong, B. Li, and C. W. Qiu, Full Control and Manipulation of Heat Signatures: Cloaking, Camouflage and Thermal Metamaterials, Adv. Mater. 26, 1731 (2014).

[9] H. Xu, X. Shi, F. Gao, H. Sun, and B. Zhang, Ultrathin Three-Dimensional Thermal Cloak, Phys. Rev. Lett. 112, 054301 (2014).

[10] X. Zheng et al. Ultralight, Ultrastiff Mechanical Metamaterials, Science 344, 1373 (2014).

[11] J. L. Silverberg, A. A. Evans, L. McLeod, R. C. Hayward, T. Hull, C. D. Santangelo, and I. Cohen, Using Origami Design Principles to Fold Reprogrammable Mechanical Metamaterials, Science 345, 647 (2014).

[12] C. Coulais, D. Sounas, and A. Alù, Static Non-Reciprocity in Mechanical Metamaterials, Nature (London) 542, 461 (2017). 
[13] Y. Wu, Y. Lai, and Z. Q. Zhang, Elastic Metamaterials with Simultaneously Negative Effective Shear Modulus and Mass Density, Phys. Rev. Lett. 107, 105506 (2011).

[14] S. Brûlé, E. H. Javelaud, S. Enoch, and S. Guenneau, Experiments on Seismic Metamaterials: Molding. Surface Waves, Phys. Rev. Lett. 112, 133901 (2014).

[15] P. Wang, F. Casadei, S. Shan, J. C. Weaver, and K. Bertoldi, Harnessing Buckling to Design Tunable Locally Resonant Acoustic Metamaterials, Phys. Rev. Lett. 113, 014301 (2014).

[16] R. Zhu, X. N. Liu, G. K. Hu, C. T. Sun, and G. L. Huang, Negative Refraction of Elastic Waves at the DeepSubwavelength Scale in a Single-Phase Metamaterial, Nat. Commun. 5, 5510 (2014).

[17] Y. Liu, X. Su, and C. T. Sun, Broadband Elastic Metamaterial with Single Negativity by Mimicking Lattice Systems, J. Mech. Phys. Solids 74, 158 (2015).

[18] Y. Lai, Y. Wu, P. Sheng, and Z. Q. Zhang, Hybrid Elastic Solids, Nat. Mater. 10, 620 (2011).

[19] T. Bückmann, M. Thiel, M. Kadic, R. Schittny, and M. Wegener, An Elasto-Mechanical Unfeelability Cloak Made of Pentamode Metamaterials, Nat. Commun. 5, 4130 (2014).

[20] G. Ma, C. Fu, G. Wang, P. Del Hougne, J. Christensen, Y. Lai, and P. Sheng, Polarization Bandgaps and Fluid-like Elasticity in Fully Solid Elastic Metamaterials, Nat. Commun. 7, 13536 (2016).

[21] J. M. Kweun, H. J. Lee, J. M. Oh, H. M. Seung, and Y. Y. Kim, Transmodal Fabry-Pérot Resonance: Theory and Realization with Elastic Metamaterials, Phys. Rev. Lett. 118, 205901 (2017).

[22] M. Farhat, S. Guenneau, and S. Enoch, Ultrabroadband Elastic Cloaking in Thin Plates, Phys. Rev. Lett. 103, 024301 (2009).

[23] N. Stenger, M. Wilhelm, and M. Wegener, Experiments on Elastic Cloaking in Thin Plates, Phys. Rev. Lett. 108, 014301 (2012).

[24] Y. Liu, Z. Liang, F. Liu, O. Diba, A. Lamb, and J. Li, Source Illusion Devices for Flexural Lamb Waves Using Elastic Metasurfaces, Phys. Rev. Lett. 119, 034301 (2017).

[25] G. W. Milton, M. Briane, and J. R. Willis, On Cloaking for Elasticity and Physical Equations with a Transformation Invariant Form, New J. Phys. 8, 248 (2006).

[26] A. N. Norris and A. L. Shuvalov, Elastic Cloaking Theory, Wave Motion 48, 525 (2011).

[27] M. Brun, S. Guenneau, and A. B. Movchan, Achieving Control of In-Plane Elastic Waves, Appl. Phys. Lett. 94, 061903 (2009).

[28] J. F. C. Yang and R. S. Lakes, Experimental Study of Micropolar and Couple Stress Elasticity in Compact Bone in Bending, J. Biomech. 15, 91 (1982).

[29] T. Frenzel, M. Kadic, and M. Wegener, Three-Dimensional Mechanical Metamaterials with a Twist, Science 358, 1072 (2017).

[30] J. R. Willis, Variational Principles for Dynamic Problems for Inhomogeneous Elastic Media, Wave Motion 3, 1 (1981).

[31] S. Nemat-Nasser and A. Srivastava, Overall Dynamic Constitutive Relations of Layered Elastic Composites, J. Mech. Phys. Solids 59, 1953 (2011).
[32] A. N. Norris, A. L. Shuvalov, and A. A. Kutsenko, Analytical Formulation of Three-Dimensional Dynamic Homogenization for Periodic Elastic Systems, Proc. R. Soc. A 468, 1629 (2012).

[33] M. B. Muhlestein, C. F. Sieck, A. Alù, and M. R. Haberman, Reciprocity, Passivity and Causality in Willis Materials, Proc. R. Soc. A 472, 20160604 (2016).

[34] Z. H. Xiang and R. W. Yao, Realizing the Willis Equations with Pre-Stresses, J. Mech. Phys. Solids 87, 1 (2016).

[35] H. Nassar, X. C. Xu, A. N. Norris, and G. L. Huang, Modulated Phononic Crystals: Non-Reciprocal Wave Propagation and Willis Materials, J. Mech. Phys. Solids 101, 10 (2017).

[36] S. Koo, C. Cho, J. H. Jeong, and N. Park, Acoustic Omni Meta-atom for Decoupled Access to All Octants of a Wave Parameter Space, Nat. Commun. 7, 13012 (2016).

[37] M. B. Muhlestein, C. F. Sieck, P. S. Wilson, and M. R. Haberman, Experimental Evidence of Willis Coupling in a One-Dimensional Effective Material Element, Nat. Commun. 8, 15625 (2017).

[38] J. Li, C. Shen, A. Díaz-Rubio, S. A. Tretyakov, and S. A. Cummer, Systematic Design and Experimental Demonstration of Bianisotropic Metasurfaces for Scattering-Free Manipulation of Acoustic Wavefronts, Nat. Commun. 9, 1342 (2018).

[39] L. Quan, Y. Ra'di, D. L. Sounas, and A. Alù, Maximum Willis Coupling in Acoustic Scatterers, Phys. Rev. Lett. 120, 254301 (2018).

[40] R. Marqués, F. Medina, and R. Rafii-El-Idrissi, Role of Bianisotropy in Negative Permeability and Left-Handed Metamaterials, Phys. Rev. B 65, 144440 (2002).

[41] J. K. Gansel, M. Thiel, M. S. Rill, M. Decker, K. Bade, V. Saile, G. von Freymann, S. Linden, and M. Wegener, Gold Helix Photonic Metamaterial as Broadband Circular Polarizer, Science 325, 1513 (2009).

[42] S. Zhang, Y. S. Park, J. Li, X. Lu, W. Zhang, and X. Zhang, Negative Refractive Index in Chiral Metamaterials, Phys. Rev. Lett. 102, 023901 (2009).

[43] E. Plum, X. X. Liu, V. A. Fedotov, Y. Chen, D. P. Tsai, and N. I. Zheludev, Metamaterials: Optical Activity without Chirality, Phys. Rev. Lett. 102, 113902 (2009).

[44] K. F. Graff, Wave Motion in Elastic Solids (Dover publications, New York, 2012).

[45] A. H. Nayfeh, Wave Propagation in Layered Anisotropic Media: With Application to Composites (Elsevier, Amsterdam, 1995).

[46] A. Diatta, Y. Achaoui, S. Brûlé, S. Enoch, and S. Guenneau, Control of Rayleigh-like Waves in Thick Plate Willis Metamaterials, AIP Adv. 6, 121707 (2016).

[47] Z. Su and L. Ye, Identification of Damage Using Lamb Waves: From Fundamentals to Applications (Springer Science \& Business Media, Berlin, 2009).

[48] T. Wang, X. Mu, P. Kropelnicki, A. B. Randles, and C. Lee, Viscosity and Density Decoupling Method Using a Higher Order Lamb Wave Sensor, J. Micromech. Microeng. 24, 075002 (2014).

[49] A. N. Norris, Flexural Waves on Narrow Plates, J. Acoust. Soc. Am. 113, 2647 (2003).

[50] See Supplemental Material at http://link.aps.org/ supplemental/10.1103/PhysRevX.9.011040 for the band 
structure of the Willis material, effects of loss on the unidirectional zero reflection, details for testing the 3D printing material parameters, and the similarity between platelike and beamlike structures.

[51] X. Wang, Acoustical Mechanism for the Extraordinary Sound Transmission through Subwavelength Apertures, Appl. Phys. Lett. 96, 134104 (2010).

[52] J. Gear, F. Liu, S. T. Chu, S. Rotter, and J. Li, Parity-Time Symmetry from Stacking Purely Dielectric and Magnetic Slabs, Phys. Rev. A 91, 033825 (2015).

[53] J. Gear, Y. Sun, S. Xiao, L. Zhang, R. Fitzgerald, S. Rotter, H. Chen, and J. Li, Unidirectional Zero Reflection as Gauged Parity-Time Symmetry, New J. Phys. 19, 123041 (2017).

[54] A. Ba, A. Kovalenko, C. Aristégui, O. Mondain-Monval, and T. Brunet, Soft Porous Silicone Rubbers with Ultra-Low Sound Speeds in Acoustic Metamaterials, Sci. Rep. 7, 40106 (2017).

[55] W. Chen, Ş. K. Özdemir, G. Zhao, J. Wiersig, and L. Yang, Exceptional Points Enhance Sensing in an Optical Microcavity, Nature (London) 548, 192 (2017).

[56] H. Hodaei, A. U. Hassan, S. Wittek, H. Garcia-Gracia, R. El-Ganainy, D. N. Christodoulides, and M. Khajavikhan,
Enhanced Sensitivity at Higher-Order Exceptional Points, Nature (London) 548, 187 (2017).

[57] T. Liu, X. Zhu, F. Chen, S. Liang, and J. Zhu, Unidirectional Wave vector Manipulation in Two-Dimensional Space with an All Passive Acoustic Parity-Time-Symmetric Metamaterials Crystal, Phys. Rev. Lett. 120, 124502 (2018).

[58] S. Brule, S. Enoch, and S. Guenneau, Experimental Evidence of Auxetic Features in Seismic Metamaterials: Ellipticity of Seismic Rayleigh Waves for Subsurface Architectured Ground with Holes, arXiv:1809.05841.

[59] X. Su, Z. Lu, and A. N. Norris, Elastic Metasurfaces for Splitting SV-and P-Waves in Elastic Solids, J. Appl. Phys. 123, 091701 (2018).

[60] Y. Ra'di, D. L. Sounas, and A. Alù, Metagratings: Beyond the Limits of Graded Metasurfaces for Wave Front Control, Phys. Rev. Lett. 119, 067404 (2017).

[61] J. Li, A. Díaz-Rubio, C. Shen, Z. Jia, S. Tretyakov, and S. A. Cummer, Highly Efficient Generation of Angular Momentum with Cylindrical Bianisotropic Metasurfaces, Phys. Rev. Applied 11, 024016 (2019).

[62] R. Craster, A. Diatta, S. Guenneau, and H. Hutridurga, Some Results in Near-Cloaking for Elasticity Systems, arXiv:1803.01360. 